\title{
Descripción de estudios de autopsias COVID-19: una revisión de alcance. Noviembre 2019 - diciembre 2020.
}

Description of COVID-19 autopsy studies: a scoping review. November 2019 - December 2020

Alisson Nohelia Lanza Duarte 1

https://orcid.org/oooo-0o01-9359-3957

Jackeline Alger2

https://orcid.org/oooo-00o1-9244-0668

César Augusto Durán López ${ }^{3}$

https://orcid.org/oooo-0oo2-9464-7889

${ }^{1}$ Universidad Nacional Autónoma de Honduras. ${ }^{2}$ Universidad Nacional Autónoma de Honduras, Unidad de Investigación Cientifica, Facultad de Ciencias Médicas. Hospital Escuela, Departamento de Laboratorio Clínico. ${ }^{3}$ Universidad Nacional Autónoma de Honduras, Departamento de Patología, Facultad de Ciencias Médicas.

\section{PALABRAS CLAVE}

Autopsia, Cambios post mortem, Hallazgos incidentales, Infecciones por coronavirus, COVID-19.

\section{KEYWORDS}

Autopsy, Post mortem changes, Incidental findings, Coronavirus infections, COVID-19.

\section{CITAR COMO}

Lanza-Duarte AN, Alger J, Durán-López CA. Descripción de Estudios de Autopsias COVID-19:

Una Revisión de Alcance.Noviembre 2019 diciembre 2020 Rev. cienc. forenses Honduras.

2021; 7(2): 3-22.

doi:10.5377/rcfh.v7i2.13171

\section{AUTOR PARA CORRESPONDENCIA}

Alisson Lanza: alis.moy@gmail.com

\section{HISTORIA DEL ARTÍCULO}

Recepción: 14 -7- 2021

Aprobación: 25 -10- 2021

\section{DOI}

https://doi.org/10.5377/rcfh.v7i2.13171

DECLARACIÓN DE RELACIONES Y ACTIVIDADES FINANCIERAS $Y$ CONFLICTOS DE INTERÉS

Ninguna

\section{RESUMEN}

Antecedentes: las autopsias son esenciales para investigar las enfermedades emergentes ya que contribuyen a comprender su fisiopatología y fundamentar el tratamiento. Objetivo: Describir las publicaciones sobre autopsias de casos COVID-19 en el marco de la pandemia, 2019-2020. Metodología: Revisión de alcance. Periodo: publicaciones de noviembre 2019 a diciembre 2020. Términos utilizados: autopsia, cambios post mortem, hallazgos incidentales, infecciones por coronavirus, en sujetos humanos, en español e inglés; en bases de datos Research4Life, PubMed, LILACS, Researchgate. Se utilizó el estándar PRISMA. El protocolo fue aprobado por la Unidad de Investigación Científica de la Facultad de Ciencias Médicas, Universidad Nacional Autónoma de Honduras. Resultados: de un total de 320 artículos identificados, se seleccionaron 42, correspondientes a 582 autopsias, todos en el idioma inglés. De los 42 artículos, los dos países con más publicaciones 


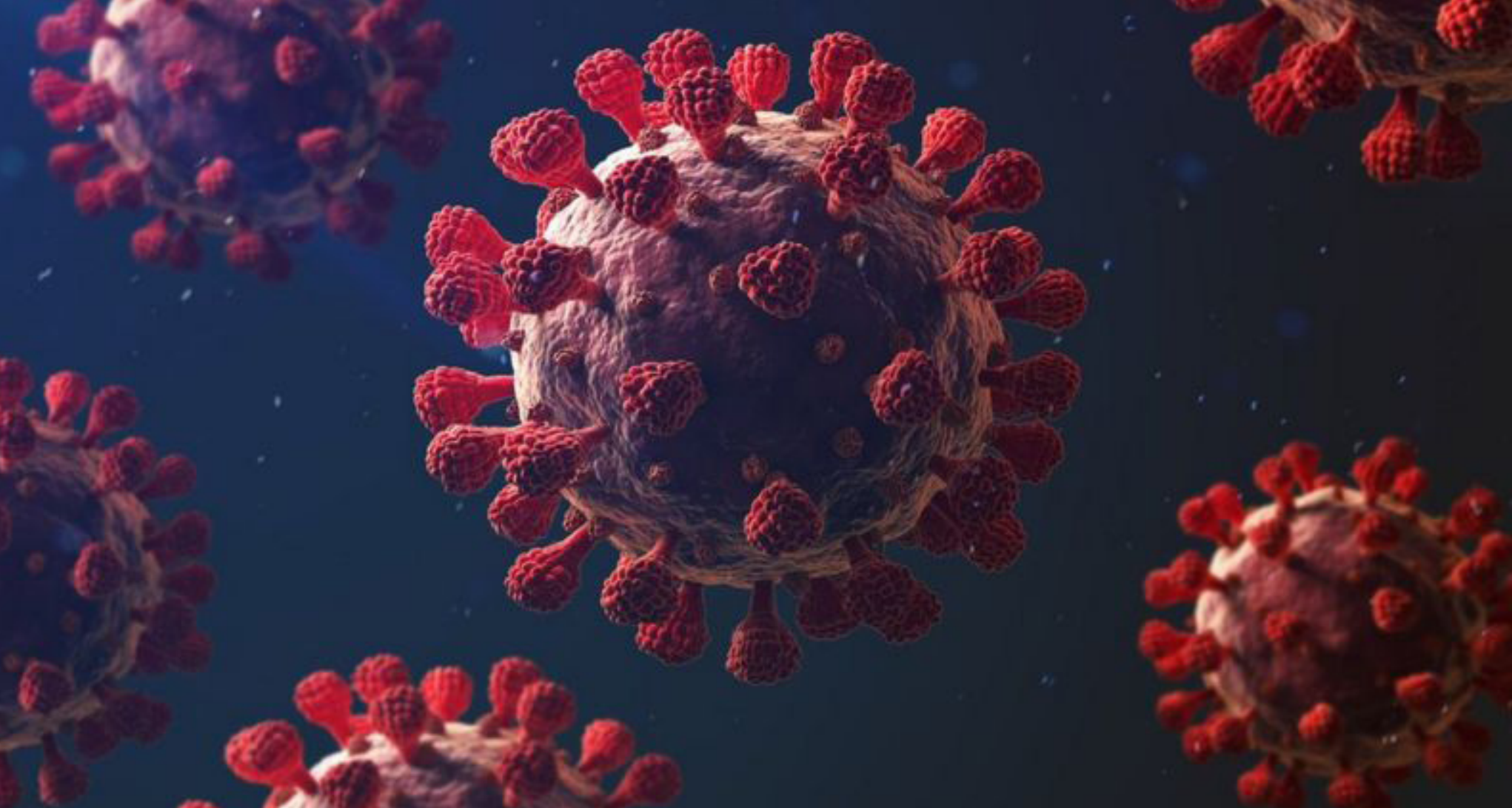

fueron USA 16 y Alemania 5. En 27 el rango de autopsias reportadas fue 1-10. En 17 las medidas de bioseguridad implementadas no fueron consignadas y en 13 no fueron especificadas. En 19, no se describe si las autopsias fueron completas o parciales. Las autopsias completas se reportaron en 14 publicaciones, parciales en 5 y autopsias completas y parciales en 4 . En 11 publicaciones no se describieron los hallazgos macroscópicos; en los 42 trabajos se examinaron los tejidos con microscopio óptico, en 28 se utilizaron marcadores inmunohistoquímicos y en 7 microscopio electrónico. El órgano descrito principalmente fue pulmón en 40 estudios. Conclusiones e implicaciones: son necesarias más publicaciones reportando series de autopsias completas, retomar la importancia del estudio de todos los órganos y la determinación de las causas de muerte por COVID-19. A diciembre de 2020, en América Latina no se había publicado estudios sobre autopsias. Las implicaciones para el manejo de casos y políticas públicas demuestran que se debe invertir en la realización de autopsias incluyendo los estudios histopatológicos que se requieran.

\section{ABSTRACT}

Background: Autopsies are essential to investigate emerging diseases because they contribute to the understanding of their pathophysiology and support their treatment. Objective: To describe the publications on autopsies of COVID-19 cases in the context of the pandemic, 2019-2020. Methodology: Scoping review. Period: November 2019-December 2020. Terms used: autopsy, post-mortem changes, incidental findings, Coronavirus infections in human subjects, in Spanish and English, in Research4Life, PubMed, LILACS, Researchgate. The PRISMA standard was used. The Research Scientific Unit, Faculty of Medical Sciences, Universidad Nacional Autónoma de Honduras, approved the protocol. Results: From a total of 320 articles identified, 42 were selected, corresponding to 582 autopsies, all in the English language. Of the 42 articles, the two countries with the most publications were USA 16 and Germany 5. In 27, the range of reported autopsies was 1-10; in 17, the biosecurity measures implemented were not recorded and in 13, they were not specified. In 19, the size of the autopsy's 
sample was not described. Complete autopsies were described in 14, complete and partial autopsies in 5 and partial autopsies in 4 . In 11, the macroscopic findings were not described; in 42 the tissues were examined with a light microscope, in 28 immunohistochemical techniques were used, and in 7, electron microscope. The most studied organ was the lung in 40 studies. Conclusions and implications: More publications are needed reporting series of complete autopsy, resuming the importance of the study of all organs, and the determination of the causes of death from COVID-19. As of December 2020, no autopsy studies had been published in Latin America. The implications for case management and public policies demonstrate that investment should be made in autopsies, including histopathological studies that are required.

\section{INTRODUCCIÓN}

La enfermedad por coronavirus (COVID-19) fue notificada por primera vez en Wuhan, China, el 31 de diciembre de 2019. La familia de los coronavirus $(\mathrm{CoV})$ son agentes de zoonosis que pueden causar desde resfriado común hasta afecciones más graves. El 13 de marzo del 2020 la Organización Mundial de la Salud (OMS) declaró como pandemia a la nueva enfermedad $^{1,2}$. Aproximadamente dieciséis meses después, a julio de 2021, la enfermedad por el nuevo coronavirus ha afectado a $182,651,733$ personas a nivel mundial, con 3,995,824 fatalidades ${ }^{3}$. Para la

\section{Las autopsias y el examen} microscópico de las muestras de tejiclo desempeñan un papel fundamental en el diagnóstico y conocimiento de la fisiopatología de cualquier enfermedad nueva misma fecha, en Honduras, se han informado 262,760 casos confirmados y ha cobrado 7,005 vidas ${ }^{4}$. Hasta el momento existe conocimiento extenso, pero desintegrado sobre el virus, con vacunas en evaluación siendo distribuidas $\mathrm{y}$ aplicadas, sin tratamiento farmacológico específico y comprensión limitada de la reinfección y duración de la inmunidad haciéndose notoria la importancia de seguir investigando.

\section{W \\ En la mayoría de los países de América Latina no se cuenta con los medios necesarios para la realización de aułopsias, por lo que el diagnostico se confirma mediante exámenes de laboratorio}

Las autopsias y el examen microscópico de las muestras de tejido desempeñan un papel fundamental en el diagnóstico y conocimiento de la fisiopatología de cualquier enfermedad nueva ${ }^{5}$; sin embargo, los exámenes post mortem de casos confirmados de la COVID-19 han sido relativamente pocos por las preocupaciones asociadas al riesgo potencial de contagio. En la mayoría de los países de América Latina no se cuenta con los medios necesarios para la realización de autopsias, por lo que el diagnóstico se confirma mediante exámenes de laboratorio y se procede a la entrega del cadáver para su respectiva inhumación/cremación. En Honduras en general se recomienda no realizar autopsia de personas fallecidas por COVID-19, excepto por indicaciones clínicas fundamentadas en cuyo caso el procedimiento se realiza de forma parcial tomando muestras de los órganos de interés ${ }^{6,7}$. El presente trabajo se realizó con el objetivo de describir las publicaciones sobre autopsias de casos 
COVID-19 en el marco de la pandemia, en el periodo de noviembre 2019 a diciembre 2020.

\section{METODOLOGÍA}

Se realizó un estudio tipo revisión de alcance (scoping review, https://guides.temple.edu/c. php?g=78618\&p=4156607). El protocolo fue revisado y aprobado por la Unidad de Investigación Científica (UIC) de la Facultad de Ciencias Médicas, Universida Nacional Autónoma de Honduras (UNAH), en noviembre 2020. Se aplicaron los siguientes criterios de búsqueda: artículos publicados en inglés y español desde noviembre del 2019 hasta diciembre del 2020, solo en sujetos humanos, buscados utilizando las plataformas:

$\rightarrow$ Hinari: https://www.who.int/hinari/es/

$\rightarrow$ PubMed: https://pubmed.ncbi.nlm.nih.gov/

$\rightarrow$ LILACS: https://lilacs.bvsalud.org/en/

$\rightarrow$ Researchgate.net: https://www.researchgate.net/

Para la búsqueda se utilizaron las palabras clave confirmadas en descriptores DeCS en inglés: incidental findings, post mortem changes, autopsy, coronavirus infections y las siguientes combinaciones: incidental findings "AND" post mortem changes "AND" coronavirus infections; incidental findings "AND" autopsy "AND" coronavirus infections. En español las palabras clave confirmadas en descriptores DeCS fueron autopsia, cambios post mortem, hallazgos incidentales, infecciones por coronavirus y las siguientes combinaciones: hallazgos incidentales "Y" cambios post mortem " $\mathrm{Y}$ " infecciones coronavirus; hallazgos incidentales "Y" autopsia "Y" infecciones coronavirus. En algunas plataformas, al introducir los términos en inglés, identificaron artículos en otros idiomas (PubMed, LILACS). Se identificaron y eliminaron los artículos duplicados. Se realizó un tamizaje inicial por título y resumen seguido del tamizaje por texto completo cuando fue necesario. Se excluyeron los estudios de biopsias, trabajos sobre hallazgos post mortem en pacientes sin PCRRT que confirmara la infección por SARS-CoV-2, publicaciones en otros idiomas y estudios en animales. Se identificó un total de 320 artículos, eliminándose 36 artículos duplicados y 241 por varias razones (figura 1), quedando un total de 42 artículos. Los artículos seleccionados se revisaron en su texto completo para extraer la información y se registró en un instrumento preparado como documento Microsoft Excel 2013® que posteriormente se cargó a la nube en Google sheet https://docs.google. com/spreadsheets/d/1MnrGAsLbZZr16E4T08TVhUgiQ5wi82oz4QBeR0o3KM/edit?usp=sharing.

Este incluyó las siguientes secciones: estudio, idioma, país de origen, número de autopsias, medidas de bioseguridad aplicadas en autopsia, número de autopsias completas y parciales, sexo, edad, causa de muerte, comorbilidad, órganos reportados, hallazgos macroscópicos y hallazgos microscópicos. Los resultados se analizaron con la elaboración de cuadros. El primero expone las características de los estudios: tipo de artículo, país de origen e idioma de publicación. En el segundo se muestra la edad y sexo de los casos estudiados, características técnicas de las autopsias (normas de bioseguridad aplicadas, abordaje del cuerpo, sea parcial o completo, técnicas para el estudio de los tejidos) y número de autopsias por publicación. En un gráfico se muestra qué órganos fueron descritos en mayor o menor cantidad, además se seleccionaron imágenes macroscópicas y microfotografías representativas de los efectos de SARS-CoV-2 en el organismo. 


\section{RESULTADOS}

Se analizaron 42 artículos para la revisión con una totalidad de 582 autopsias descritas. El tipo, idioma y país donde se realizó el estudio se muestra en el cuadro

1. Respecto al tipo de autopsia realizada, completa o parcial, de los 42 estudios 19 no lo describieron, 14 mencionaron que fueron autopsias completas, 5 refirieron la realización de autopsias parciales y 4 reportaron tanto autopsias completas como parciales. Se encontró que en 17 estudios las medidas de bioseguridad implementadas en las autopsias no fueron consignadas. En 13 no fueron específicas y en 12 indicaron seguir las normas implementadas por instituciones reconocidas (cuadro 2). En cuanto a la edad reportada de los casos autopsiados en los 42 estudios, en 11 se determinó la edad media y no individualmente por el tamaño de su muestra. Por lo tanto, solo se incluyeron 31 estudios para establecer la frecuencia de rangos etarios en las publicaciones.

Con relación al sexo, un estudio no reveló el sexo de los casos involucrados, por lo que el análisis se realizó con los 41 restantes. En 11 de los 42 estudios no se describieron hallazgos macroscópicos de ningún órgano. En todos los estudios se realizó examen por microscopía óptica aplicando la tinción de rutina hematoxilina - eosina (HE), en 28 trabajos se reportó la utilización de marcadores inmunohistoquímicos y

Cuadro 1. Características de los estudios de autopsias COVID-19, noviembre 2019 - diciembre 2020, n=42.

\begin{tabular}{|c|c|}
\hline CARACTERISTICAS & $\mathbf{N}(\%)$ \\
\hline \multicolumn{2}{|l|}{ Iipo de artículo } \\
\hline Artículos originales & $27(64.3)$ \\
\hline Reportes de casos clínicos & $11(26.2)$ \\
\hline Cartas al editor & $4(9.5)$ \\
\hline \multicolumn{2}{|l|}{ País del estudio* } \\
\hline Estados Unidos de América ${ }^{8-23}$ & $16(38.0)$ \\
\hline Alemania $^{24-28}$ & $5(11.9)$ \\
\hline Italia $29-31$ & $3(7.1)$ \\
\hline Irán 32 - 34 & $3(7.1)$ \\
\hline Rumania $^{35-37}$ & $3(7.1)$ \\
\hline China $^{38,39}$ & $2(5.0)$ \\
\hline Reino Unido ${ }^{40,41}$ & $2(5.0)$ \\
\hline Suiza ${ }^{42,43}$ & $2(5.0)$ \\
\hline Brasil $^{44}$ & $1(2.3)$ \\
\hline Japón ${ }^{45}$ & $1(2.3)$ \\
\hline Francia $^{46}$ & $1(2.3)$ \\
\hline Dinamarca $^{47}$ & $1(2.3)$ \\
\hline España $a^{48}$ & $1(2.3)$ \\
\hline Rusia $^{49}$ & $1(2.3)$ \\
\hline \multicolumn{2}{|l|}{ Idioma de la publicación } \\
\hline Inglés & $42(100.0)$ \\
\hline Español & $0(0.0)$ \\
\hline
\end{tabular}

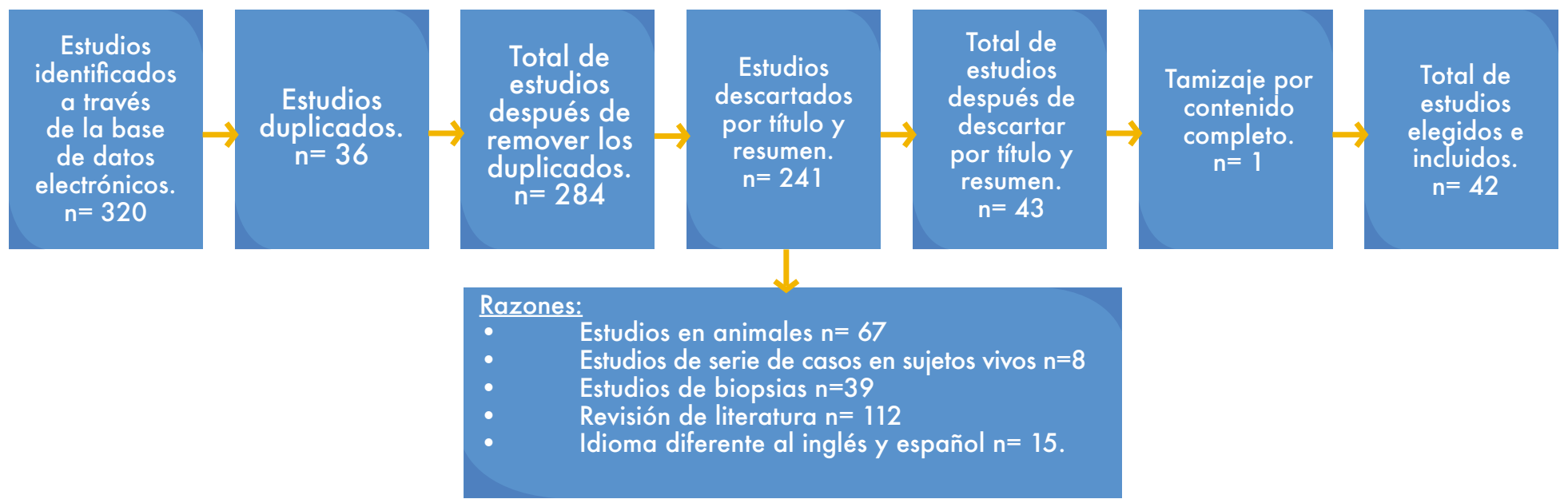

Figura 1. Diagrama de flujo que ilustra el proceso de búsqueda y selección de artículos, estudios de autopsias COVID-19, noviembre 2019 - diciembre 2020 
Cuadro 2. Edad y sexo de los casos, características técnicas de las autopsias reportadas y número de autopsias por publicación.

\begin{tabular}{|c|c|}
\hline CARACTERISTICAS & $\mathbf{N}(\%)$ \\
\hline \multicolumn{2}{|l|}{ Edad (años), N=242 cutopsias * } \\
\hline $20-39$ & $17(7.0)$ \\
\hline $40-59$ & $37(15.0)$ \\
\hline $60-79$ & $106(44.0)$ \\
\hline $80-99$ & $82(34.0)$ \\
\hline Dato no recuperable & $338(58.3)$ \\
\hline \multicolumn{2}{|l|}{ Sexo, $N=515$ autopsias * } \\
\hline Femenino & $181(35.0)$ \\
\hline Masculino & $334(65.0)$ \\
\hline Dato no recuperable & $67(13.0)$ \\
\hline \multirow{2}{*}{\multicolumn{2}{|c|}{$\begin{array}{l}\text { Normas de bioseguridad aplicadas } \\
\text { en las autopsias, N=42 estudios }\end{array}$}} \\
\hline & \\
\hline No fueron consignadas & $17(40.4)$ \\
\hline No fueron especificas * * & $13(31.0)$ \\
\hline $\mathrm{CDC}$ & $4(10.0)$ \\
\hline Bioseguridad para patógenos BSL3 & $2(4.7)$ \\
\hline College of American Pathologists & $2(4.7)$ \\
\hline Royal College of Pathologists & $1(2.3)$ \\
\hline Sociedad Española de Patología & $1(2.3)$ \\
\hline Sociedad Danesa de Patología & $1(2.3)$ \\
\hline Recomendaciones Italianas & $1(2.3)$ \\
\hline \multicolumn{2}{|l|}{ Autopsia completa/parcial, } \\
\hline \multicolumn{2}{|l|}{$\underline{N}=42$ estudios } \\
\hline No lo describen & $19(45.0)$ \\
\hline Autopsias completas *** & $14(33.0)$ \\
\hline Autopsias parciales & $5(12.0)$ \\
\hline Autopsias completas y parciales & $4(10.0)$ \\
\hline \multicolumn{2}{|l|}{ Estudio de teildo, N=42 estudios } \\
\hline $\begin{array}{l}\text { Descripción de hallazgos } \\
\text { macroscópicos }\end{array}$ & $31(73.8)$ \\
\hline Examen por microscopia óptica & $42(100.0)$ \\
\hline $\begin{array}{l}\text { Aplicación de marcadores } \\
\text { inmunohistoquímicos. }\end{array}$ & $28(66.6)$ \\
\hline $\begin{array}{l}\text { Examen por microscopia } \\
\text { electrónica }\end{array}$ & $7(16.6)$ \\
\hline \multicolumn{2}{|l|}{ Número de autopsias realizadas } \\
\hline \multicolumn{2}{|l|}{ por estudio, N=42 estudios } \\
\hline $1-10$ & $27(64.2)$ \\
\hline $11-20$ & $6(14.2)$ \\
\hline $21-30$ & $1(2.3)$ \\
\hline $31-40$ & $5(12.0)$ \\
\hline $41-60$ & $1(2.3)$ \\
\hline $61-80$ & $2(5.0)$ \\
\hline
\end{tabular}

en 7 se utilizó microscopia electrónica (cuadro 2). Las diez comorbilidades más frecuentes que presentaron los casos autopsiados fueron: hipertensión arterial 294, diabetes mellitus tipo II 170, enfermedad arterial coronaria 104, lesión renal crónica 98, obesidad 71, insuficiencia cardiaca 64, cardiopatía isquémica 59, enfermedad pulmonar obstructiva crónica 55, fibrilación auricular 36, demencia 34. En 11 casos de autopsias no se encontraron comorbilidades y en 17 no se consignaron. Las 5 primeras causas de muerte en 29 estudios, escritas textualmente fueron: neumonía 142, SARS-CoV-2 37, insuficiencia respiratoria 22, infarto agudo de miocardio 13 y síndrome de distrés respiratorio agudo del adulto (SDRA) 11. En dos casos, las causas de muerte fueron desconocidas. Las causas de muerte no fueron descritas en 13 estudios y en seis la descripción no fue completa, en total 267 autopsias no tenían causa de muerte. Los órganos reportados con mayor frecuencia fueron: pulmón 40, corazón 30, hígado y riñones con 24 cada uno (ver figura 2). Los efectos del SARS-CoV-2 descritos principalmente fueron pulmonares caracterizados por diversas etapas de daño alveolar difuso (DAD), las fases: exudativa con formación de membranas hialinas, proliferativa con hiperplasia de neumocitos tipo II y de reparación temprana con hiperplasia de células fusiformes intersticiales y organización intraalveolar. En la figura 3 se presentan algunas imágenes extraídas de las publicaciones para ilustrar las alteraciones morfológicas que produce la infección por SARSCoV-2. La selección destaca las alteraciones en los pulmones como: vasos trombosados, la evolución

\footnotetext{
*Para determinar el rango edad, se utilizó la información de 31/42 estudios (242 autopsias), ya que en 11 solo se registró la edad media debido al tamaño muestral. Para determinar el sexo se utilizó la información de 41/42 estudios (515 autopsias), ya que en uno el sexo no se registró. ** Solo mencionaron implementos de equipo de protección personal (EPP) y salas de presión negativa. ***Algunas obviaron el sistema nervioso central (SNC) para disminuir la exposición de partículas. Abreviaturas: CDC=Centros para el Control y la Prevención de Enfermedades de los Estados Unidos. BSL3=Laboratorio de contención de nivel 3 de seguridad biológica.
} 
40

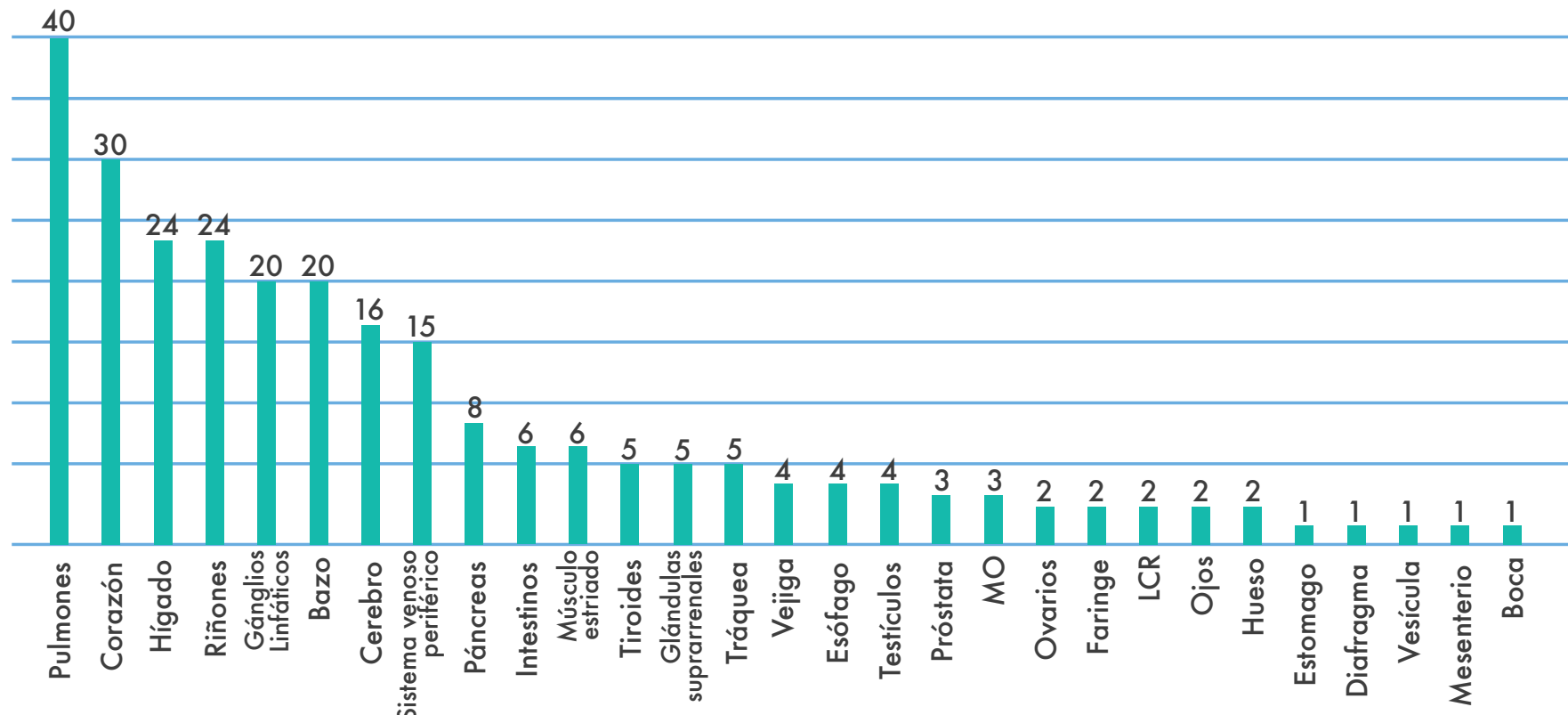

Figura 2. Estructuras descritas en los estudios de autopsias COVID-19, N= 42 noviembre 2019 - diciembre 2020. MO: médula ósea. LCR: líquido cefalorraquídeo.

del daño alveolar difuso desde su etapa aguda hasta la fibrosis y el uso de inmunohistoquímica para determinar la presencia del virus en el tejido.

\section{DISCUSIÓN}

En esta revisión de alcance se analizaron 42 estudios con un total de 582 autopsias reportadas. Todos en el idioma inglés, realizados principalmente en Estados Unidos de Norte América seguidos por Alemania, Italia, Rumanía, Irán, China y Reino Unido. Al inicio de la pandemia, las autopsias descritas fueron en su mayoría de China, lugar donde emergió el primer brote $^{52,53}$. Al diseminarse el virus, la preocupación a nivel mundial hizo que los países desarrollados enfocaran sus recursos en el estudio de la enfermedad, lo que podría explicar la distribución geográfica de los estudios analizados. Que no hayan sido encontrados estudios en Latinoamérica, podría indicar la falta de inversión en salud, en particular la destinada a generar conocimiento que dé respuesta a los problemas de la población ${ }^{54-56}$. En casi la mitad de las publicaciones estudiadas no se describió si la autopsia fue completa o parcial, omitiendo un aspecto importante de la metodología de esos trabajos, que inducen al lector a asumir que los procedimientos fueron completos y los hallazgos descritos son únicos o los más relevantes, dejando un margen para la caracterización incompleta de la patología. La importancia de la autopsia en la pandemia se debe al aporte de información para comprender la enfermedad con fines preventivos y terapéuticos. Las publicaciones deben ser detalladas en sus aspectos metodológicos para evitar pasar por alto información necesaria para comprender los mecanismos que conducen al desenlace fatal ${ }^{57-59}$. Para la descripción de los hallazgos tisulares, en todas las publicaciones se utilizó la microscopia óptica, en poco más de la mitad (28) de las mismas aplicaron marcadores inmunohistoquímicos y una minoría (7) microscopia electrónica. El uso de estas técnicas podría deberse a sus diseños metodológicos influido por la disponibilidad de recursos o los objetivos en diferentes etapas para el estudio de la enfermedad, iniciando con trabajos de caracterización puramente morfológica, en la mayoría de los casos complementados con el 
inmunomarcaje de elementos virales dentro de las células, para después continuar con publicaciones sobre utilización de pruebas moleculares. La microscopia electrónica es el recurso más costoso y el menos utilizado en las publicaciones estudiadas ${ }^{15,60-62}$. En la mayoría de los estudios se describió el análisis de los pulmones, seguido del corazón, hígado y riñones. Estos resultados son similares a lo informado en otras publicaciones, las cuales también hacen referencia a que los hallazgos de algunos órganos no fueron descritos completamente y se omitió la descripción de otros, aun cuando las autopsias eran completas ${ }^{63-66}$. Es recomendable que las publicaciones de autopsias sobre enfermedades nuevas sean detalladas de tal manera que se describan todos los órganos, aun cuando no muestren alteraciones o muestren cambios que aparentemente no estén relacionados con la enfermedad. Esto puede ayudar a establecer relaciones de causalidad entre el agente etiológico, la fisiopatología y los cambios estructurales en órganos y sistemas para tener una caracterización certera y completa de la enfermedad ${ }^{67}$. El presente trabajo reveló que las publicaciones que informaron la edad de los pacientes (equivalente a 268 casos), 188 eran personas mayores de 60 años, similar a lo expuesto en otros artículos acerca de la vulnerabilidad para enfermar gravemente a partir de los 50 años y de forma letal después los 60 años ${ }^{68,69}$. En lo referente al sexo de los autopsiados, de 41 estudios, 334 eran hombres y 181 mujeres, estadística que concuerda con las publicaciones realizadas por la OMS donde la mortalidad es mayor en hombres $(51 \% \text { frente al } 47 \% \text {, respectivamente })^{68}$. En cuanto a las comorbilidades reportadas, no se describieron en 17 casos de autopsias y en 11 casos no había antecedentes patológicos; En el resto (554 autopsias) las comorbilidades más frecuentes fueron: hipertensión arterial, diabetes mellitus tipo II, lesión renal crónica, enfermedad arterial coronaria y obesidad. Salinas J et al. (México) con una muestra de 17.479 pacientes, reportó que las comorbilidades más frecuentes eran hipertensión arterial 13.5\%, obesidad, $11.6 \%$, diabetes $10.9 \%$, tabaquismo $5.35 \%$ y asma $1.8 \%{ }^{71}$. Wei-jie Guan et al. (China) estudiaron 1.590 pacientes, las comorbilidades frecuentes fueron: hipertensión 16.9\%, enfermedades cardiovasculares $3.7 \%$, enfermedades cerebrovasculares $1.9 \%$, diabetes $8.2 \%$ e infecciones por hepatitis B $1.8 \%{ }^{70}$. En Canadá, la base de datos sobre defunciones de estadísticas vitales (CVSD) en más de 9.500 muertes se identificaron $38 \%$ de casos con demencia o diagnóstico de Alzheimer, 33\% neumonía, 15\% hipertensión arterial, 13\% cardiopatía isquémica y $13 \%$ insuficiencia respiratoria ${ }^{73}$. Tomando en cuenta que este articulo incluyó estudios de varios países, la frecuencia de las comorbilidades podría variar según la región de procedencia de los trabajos. Las cinco primeras causas de muerte directa descritas en 29 estudios que las consignaron fueron: neumonía, SARS-CoV-2, insuficiencia respiratoria, infarto agudo de miocardio y SDRA. Destacando que la causa básica de muerte fueron complicaciones respiratorias y cardiacas. Similar al estudio ruso de Zayratyants $\mathrm{O}$, et al, con 2.000 autopsias que reportaron como causas más frecuentes de muerte el síndrome de distrés respiratorio agudo, embolia pulmonar, edema

Las cinco primeras causas de muerte directa descritas en 29 estudios que las consignaron fueron: neumonía, SARS-CoV-2, insuficiencia respiratoria, infarto agudo de miocardio, y síndrome de distrés respiratorio agudo del adulto. Destacando que la causa básica de muerte fueron complicaciones respiratorias y cardiacas.

Rev. cienc. forenses Honduras 10 Open Access
Volumen 7, N. ํ 2, Año 2021 (c)Autor(es)
URL: http://www.bvs.hn/RCFH/html5/ CC-BY-NC 4.0 internacional 
cerebral con síndrome de dislocación, septicemia, infarto agudo de miocardio ${ }^{65}$. En el presente trabajo de revisión 13 estudios no describían las causas de muerte y en 6 no las describieron completamente, con un total de 267 autopsias sin causa de muerte establecida. En dos estudios se recalca que las causas de muerte fueron desconocidas. Consideramos que información tan importante para el estudio de una patología nueva no debería ser omitida de las publicaciones ya que la OMS ha divulgado orientaciones para determinar la COVID-19 como causa de muerte $^{74}$. De las publicaciones incluidas, diecisiete no describieron las medidas de bioseguridad aplicadas en las autopsias $\mathrm{y}$ en trece no
Las escasas publicaciones sobre autopsias de casos confirmados COVID-19 en América Latina y otras regiones, puede deberse a la ausencia de condiciones apropiadas para su ejecución autopsia aceptado de forma general, así como también deuna propuesta de protocoloadaptada las condiciones regionales, nacionales y locales ${ }^{78,79}$. También es posible que, a pesar de tener las condiciones ideales, sería difícil revertir la tendencia persistente y a la baja de no ejecutar o solicitar autopsias hospitalarias ${ }^{80}$. Es contradictorio que hoy, cuando más se necesita de esta herramienta, se ha olvidado que "Mortui vivos docent" (los muertos enseñan a los vivos). Esta revisión de alcance está limitada por la búsqueda de artículos incluyendo solamente dos idiomas, inglés y español, y la utilización de solamente cuatro buscadores bibliográficos. Adicionalmente, se limitó el 99 tiempo de búsqueda al primer especificaron cuales, lo que podría denotar la falta de aceptación de un protocolo único y reproducible que describa la forma segura para realizar autopsias en cuerpos con infección por SARS-CoV-2, como se propone en diferentes estudios ${ }^{55,56,75-77}$. Concluimos que son necesarias más publicaciones reportando series de autopsias con determinación de las causas de muerte en la COVID-19 como parte de la caracterización de la enfermedad, también es importante establecer la asociación estadística entre el fallecimiento y complicaciones como: la sobreinfección bacteriana, eventos coronarios y secuelas del daño pulmonar. Las escasas publicaciones sobre autopsias de casos confirmados de COVID-19 en América Latina y otras regiones, puede deberse a la ausencia de condiciones apropiadas para su ejecución y de un protocolo de año de la pandemia. Es importante que los estados incrementen la inversión en salud, especialmente en recursos humanos y logísticos destinados a la realización de autopsias con alto riesgo de contagio, con el propósito de obtener más información para la adecuada caracterización morfológica y evolutiva de la COVID-19. En Honduras únicamente dos hospitales en Tegucigalpa, capital del país, realizan autopsias: Hospital Escuela y Hospital María. El estado, a través de la Secretaria de Salud, es la dependencia encargada de hacer efectiva la observancia y aplicación de la Ley de Autopsia Médica Obligatoria ${ }^{81}$, marco legal que debería ser utilizado para realización de autopsias en casos de infección por SARS-CoV-2, siempre y cuando se proporcionen los recursos necesarios para prevenir el contagio entre el personal médico y técnico.
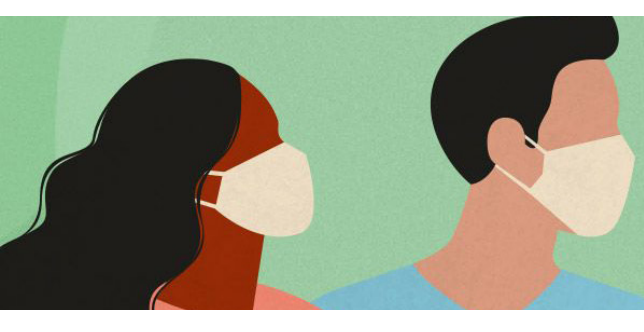

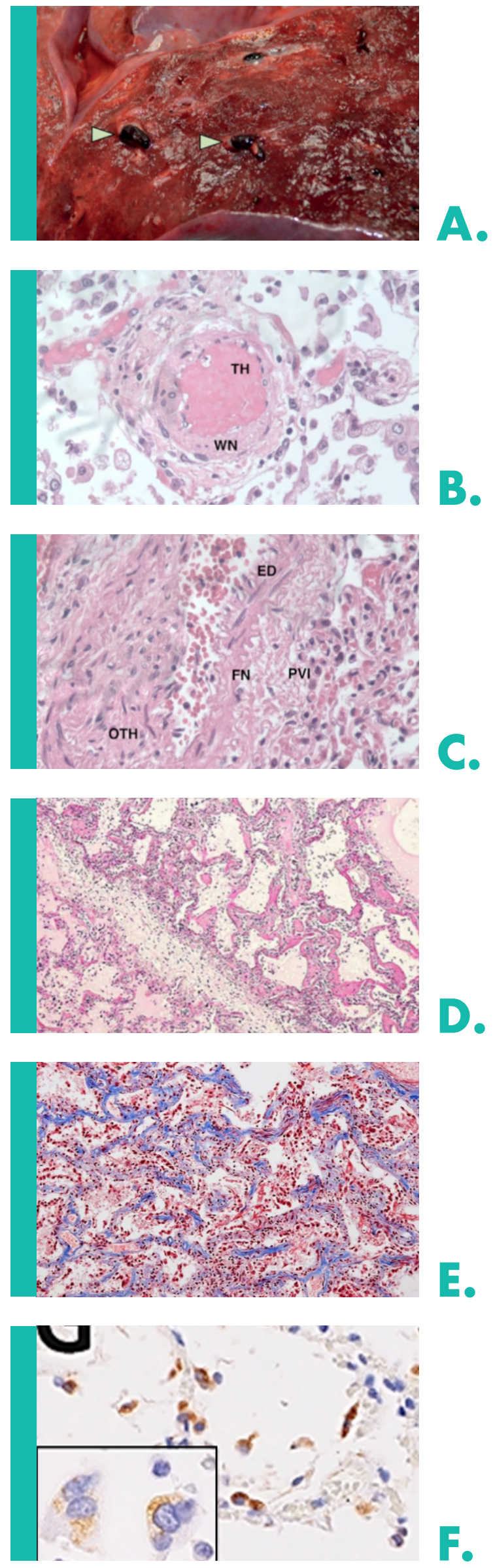

Figura 3. Muestra imagen macroscópica y microfotografías que ilustran el daño pulmonar encontrado en los fallecidos por COVID-19, estudios de autopsias COVID-19, noviembre 2019 - diciembre 2020. Los textos de las fotos se adaptaron y se agregaron comentarios explicativos adicionales. El texto entre comillas se extrajo de las referencias origen de las imágenes. A. "Pulmones con embolismo (cabezas de flechas)"14. B. "Trombo (TH) en etapa temprana y pared arterial con necrosis (WN) e infiltrado inflamatorio perivascular (HE)" 48 . Microfotografía con $23 \%$ más de brillo. C. "Trombo organizado (OTH) en la luz arłerial, descamación endotelial (ED), necrosis fibrinoide de la pared vascular (FN) e infiltrado de linfocitos en el tejido perivascular (PVI) (HE, magnificación $400 x)^{\prime \prime 48}$. Microfotografía con $25 \%$ más de brillo. D. "Fase exudativa aguda del daño alveolar difuso $(100 x)^{28 \prime \prime}$. Microfotografía recortada y reorientada del original. Las membranas hialinas están presentes en casi todos los alveolos visualizándose como material rosado y grueso en coloración Hematoxilina \& Eosina (HE). E. "Fibrosis densa (coloración tricrómica, 200x)"28. Tejido pulmonar teñido con coloración especial para resaltar el depósito de colágena en los septos alveolares (azul). F. Inmunomarcaje positivo (café) para el antígeno del SARSCoV-2 en las células epiteliales alveolares. Recuadro: Células sincitiales multinucleadas (cambio celular reactivo). Microfotografía con $20 \%$ más de nitidez ${ }^{44}$. La inmunohistoquímica es una herramienta diagnóstica que utiliza anticuerpos para identificar antígenos en la superficie o dentro de las células. Si un determinado antígeno está presente en el tejido se genera una reacción en color indicando su presencia y localización. 


\section{REFERENCIAS BIBLIOGRÁFICAS}

\section{1.}

Organización Mundial de la Salud. Coronavirus [Internet]. Washington: OPS; 2021. [citado 05 enero 2021]. Disponible en: https://www.who.int/es/healthtopics/coronavirus/coronavirus\#tab=tab_1

\section{2.}

Organización Mundial de la Salud. Cronología de la respuesta de la OMS a la COVID-19" [Internet]. Washington: OPS; 2021 [citado 05 enero 2021]. Disponible en: https://www.who.int/es/news/item/2906-2020-covidtimeline

\section{3.}

Johns Hodkins University . Center for System Science and Engineering. Distribución geográfica del brote de la enfermedad por el coronavirus (COVID-19) en el mundo [actualizado 1 julio 2021; citado 02 julio 2021]. Disponible en: http://www.bvs.hn/COVID-19/ index $2 . h t m l$

\section{4.}

Unidad de Vigilancia de Salud (HN). Estadística nacional de coronavirus COVID-19 [actualizado 1 julio 2021; citado 02 julio 2021]. Disponible en: http:// www.bvs.hn/COVID-19/

\section{5.}

Kumar V, Abbas A, Aster J. Robbins Patología Humana. 10ª ed. Barcelona: Elsevier; 2018

\section{6.}

Ministerio de Salud (PE). Directiva Sanitaria No $087-$ 2020- DIGESA/MINSA Directiva Sanitaria para el manejo de cadáveres por COVID-19. Perú: Ministerio de Salud; 2020.

\section{7.}

Secretaría de Salud (HN). Lineamientos para el manejo de cadáver por casos de COVID- 19 Tegucigalpa: SESAL; 2020.

\section{8.}

Zhou L, XuZ, Castiglione GM, Soiberman US, Eberhart CG, Duh EJ. ACE2 and TMPRSS2 are expressed on the human ocular surface, suggesting susceptibility to SARS-CoV-2 infection. Ocul Surf [Internet]. 2020 [citado 15 enero 2021];18(4):537-44. Disponible en: https://pubmed.ncbi.nlm.nih.gov/32544566/

\section{9.}

Iuga $\mathrm{AC}$, Marboe $\mathrm{CC}$, Yilmaz MM, Lefkowitch JH, Gauran C, Lagana SM. Adrenal vascular changes in COVID-19 autopsies. Arch Pathol Lab Med [Internet]. 2020. [citado 15 enero 2021]; 144(10):115960. Disponible en: https://pubmed.ncbi.nlm.nih. gov/32579380/

\section{0.}

Konopka KE, Wilson A, Myers JL. Postmortem lung findings in a patient with asthma and coronavirus disease 2019. Chest [Internet]. 2020 [citado 15 enero 2021];158(3):e99-e101. Disponible en: https:// pubmed.ncbi.nlm.nih.gov/32360729/

\section{1.}

Elsoukkary SS, Mostyka M, Dillard A, Berman DR, Ma LX, Chadburn A, et al. Autopsy findings in 32 patients with COVID-19: a single-institution experience. Pathobiology [Internet]. 2021 [citado15 enero 2021];88:56-68. Disponible en: https://www. karger.com/Article/FullText/511325 


\section{2.}

Barton LM, Duval EJ, Stroberg E, Ghosh S, Mukhopadhyay S. COVID-19 autopsies, Oklahoma, USA. Am J Clin Pathol [Internet]. 2020 [citado 15 enero 2021];153(6):725-33. Disponible en: https:// academic.oup.com/ajcp/article/153/6/725/5818922.

\section{3.}

Konopka KE, Nguyen T, Jentzen JM, Rayes O, Schmidt CJ, Wilson AM, et al. Diffuse alveolar damage (DAD) from coronavirus disease 2019 infection is morphologically indistinguishable from other causes of DAD. Histopathology [Internet]. 2020 [citado 15 enero 2021];77(4):570-8. Disponible en: https:// pubmed.ncbi.nlm.nih.gov/32542743/

\section{4.}

De Michele S, Sun Y, Yilmaz MM, Katsyv I, Salvatore M, Dzierba AL, et al. Forty postmortem examinations in COVID-19 patients: two distinct pathologic phenotypes and correlation with clinical and radiologic findings. Am J Clin Pathol [Internet]. 2020 [citado 15 enero 2021];154(6):748-60. Disponible en: https:// academic.oup.com/ajcp/article/154/6/748/5900519.

\section{5.}

Bradley B, Maioli H, Johnston R, Chaudhry I, Fink S, $\mathrm{Xu} \mathrm{H}$, et al. Histopathology and ultrastructural findings of fatal COVID-19 infections in Washington State: a case series. Lancet [Internet]. 2020 [citado 15 enero 2021];396(10247):320-32. Disponible en: https:// www.thelancet.com/journals/lancet/article/PIIS01406736(20)31305-2/fulltext

\section{6.}

RapkiewiczAV, Mai X, Carsons SE, Pittaluga S, Kleiner DE, Berger JS, et al. Megakaryocytes and plateletfibrin thrombi characterize multi-organ thrombosis at autopsy in COVID-19: a case series. EClinicalMedicine [Internet]. 2020 [citado 15 enero 2021];24:100434. Disponible en: https://www.sciencedirect.com/ science/article/pii/S2589537020301784

\section{7.}

Sekulic M, Harper H, Nezami BG, Shen DL, Sekulic SP, Koeth AT, et al. Molecular detection of SARSCoV-2 infection in FFPE samples and histopathologic findings in fatal SARS-CoV-2 cases. Am J Clin Pathol [Internet]. 2020 [citado 15 de enero 2021];154(2):190200. Disponible en: https://pubmed.ncbi.nlm.nih. gov/32451533/

\section{8.}

Bryce C, Grimes Z, Pujadas E, Ahuja S, Beasley MB, Albrecht R, et al. Pathophysiology of SARS-CoV-2: targeting of endothelial cells renders a complex disease with thrombotic microangiopathy and aberrant immune response. The Mount Sinai COVID-19 autopsy experience. MedRxiv [Preprint]. 2020 [citado 15 de enero 2021]. Disponible en: https://www. medrxiv.org/content/10.1101/2020.05.18.20099960v1

\section{9.}

Lacy JM, Brooks EG, Akers J, Armstrong D, Decker L, Gonzalez A, et al. COVID-19: postmortem diagnostic and biosafety considerations. Am J Forensic Med Pathol [Internet]. 2020 [citado 15 enero 2021];41(3):143-51. Disponible en: https://pubmed.ncbi.nlm.nih.gov/32379077/ 


\section{0.}

Santoriello D, Khairallah P, Bomback AS, Xu K, Kudose S, Batal I, et al. Postmortem kidney pathology findings in patients with COVID-19. J Am Soc Nephrol [Internet]. 2020 [citado el 15 enero 2021];31(9):215867. Disponible en: https://pubmed.ncbi.nlm.nih. gov/32727719/

\section{1.}

Buja LM, Wolf DA, Zhao B, Akkanti B, McDonald $\mathrm{M}$, Lelenwa L, et al. The emerging spectrum of cardiopulmonary pathology of the coronavirus disease 2019 (COVID-19): report of 3 autopsies from Houston, Texas, and review of autopsy findings from other United States cities. Cardiovasc Pathol [Internet]. 2020 [citado 15 enero 2021];48:107233. Disponible en: https://www.sciencedirect.com/science/article/pii/ S1054880720300375.

\section{2.}

Roden AC, Bois MC, Johnson TF, Aubry MC, Alexander MP, Hagen CE, et al. The spectrum of histopathologic findings in lungs of patients with fatal coronavirus disease 2019 (COVID-19) infection. Arch Pathol Lab Med [Internet]. 2020 [citado 15 enero 2021]. Disponible en: https://p ubmed.ncbi.nlm.nih. gov/32821902/

\section{3.}

Achua JK, Chu KY, Ibrahim E, Khodamoradi K, Delma KS, Iakymenko OA, et al Histopathology and ultrastructural findings of fatal COVID-19 infections on testis. World J Mens Health [Internet]. 2020 [citado 11 junio 2021];39(1):65-74. Disponible en: https:// n9.cl/hb8kw https://

\section{4.}

Lindner D, Fitzek A, Bräuninger H, Aleshcheva G, Edler C, Meissner K, et al. Association of cardiac infection with SARS-CoV-2 in confirmed COVID-19 autopsy cases. JAMA Cardiol [Internet]. 2020 [citado 15 enero 2021];5(11):1281-85. Disponible en: https:// pubmed.ncbi.nlm.nih.gov/32730555/ doi: 10.1001/ jamacardio.2020.3551

\section{5.}

Wichmann D, Sperhake JP, Lütgehetmann M, Steurer S, Edler C, Heinemann A, et al. Autopsy findings and venous thromboembolism in patients with COVID-19: a prospective cohort study. Ann Intern Med [Internet]. 2020 [citado 15 enero 2021];173(4):268-77. Disponible en: https://pubmed.ncbi.nlm.nih.gov/32374815/

\section{6.}

Edler C, Schröder AS, Aepfelbacher M, Fitzek A, Heinemann A, Heinrich F, et al. Dying with SARS-CoV-2 infection-an autopsy study of the first consecutive 80 cases in Hamburg, Germany. Int J Legal Med [Internet]. 2020 [citado 15 enero 2021];134(4):1275-84. Disponible en: https://pubmed. ncbi.nlm.nih.gov/32500199/

\section{7.}

Schaller T, Hirschbühl K, Burkhardt K, Braun G, Trepel M, Märkl B, et al. Postmortem examination of patients with COVID-19. JAMA [Internet]. 2020 [citado 15 enero 2021]. Disponible en: https://www. ncbi.nlm.nih.gov/pmc/articles/PMC7243161/ 


\section{8.}

Bösmüller H, Traxler S, Bitzer M, Häberle H, Raiser W, Nann D, et al. The evolution of pulmonary pathology in fatal COVID-19 disease: an autopsy study with clinical correlation. Virchows Arch [Internet]. 2020 [citado 15 enero 2021];477(3):349-57. Disponible en: https://pubmed.ncbi.nlm.nih.gov/32607684/

\section{9.}

Damiani S, Fiorentino M, de Palma A, Foschini MP, Lazzarotto T, GabrielliL, etal. Pathological postmortem findings in lungs infected with SARS-Cov 2. J Pathol [Internet]. 2020 [citado 15 enero 2021];253(1):3140. Disponible en: https://onlinelibrary.wiley.com/ doi/10.1002/path.5549.

\section{0.}

Falasca L, Nardacci R, Colombo D, Lalle E, Di Caro A, Nicastri E, et al. Postmortem findings in italian patients with COVID-19: a descriptive full autopsy study of cases with and without co-morbidities. J Infect Dis [Internet]. 2020 [citado 15 enero 2021];222(11):180715. Disponible en: https://pubmed.ncbi.nlm.nih. gov/32914853/

\section{1.}

Carsana L, Sonzogni A, Nasr A, Rossi RS, Pellegrinelli A, Zerbi $\mathrm{P}$, et al. Pulmonary post-mortem findings in a series of COVID-19 cases from northern Italy: a twocentre descriptive study. Lancet Infect Dis [Internet]. 2020 [citado 15 enero 2021]. Disponible en: https:// pubmed.ncbi.nlm.nih.gov/32526193/

\section{2.}

Sadegh Beigee F, Pourabdollah Toutkaboni M, Khalili N, Nadji SA, Dorudinia A, Rezaei M, et al. Diffuse alveolar damage and thrombotic microangiopathy are the main histopathological findings in lung tissue biopsy samples of COVID-19 patients. Pathol Res Pract [Internet]. 2020 [citado 15 enero 2021];216(10):153228. Disponible en: https://pubmed. ncbi.nlm.nih.gov/32979740/

\section{3.}

Montazer F, Divsalar F, Nokiani A, Tavirani MR, Gharab SG, Javad Moosavi SA, et al. Histopathologic findings in COVID-19 autopsies from IRAN: a comprehensive report of laboratory, chest computed tomography (CT) and morphology findings [Preprint]. 2020 [citado 15 enero 2021]. Disponible en: https:// www. authorea.com/users/328090/articles/455450histopathologic-findings-in-covid-19-autopsies-fromiran-a-comprehensive-report-of-laboratory-chestcomputed-tomography-ct-and-morphology-finding s? commit $=$ ecefd2547605e174d7457a56b12012f72 cd53884

\section{4.}

Cîrstea AE, Buzulică RL, Pirici D, Ceauşu MC, Iman RV, Gheorghe OM, et al. Histopathological findings in the advanced natural evolution of the SARS-CoV-2 infection. Rom J Morphol Embryol [Internet]. 2020 [citado 15 enero 2021];61(1):209-18. Disponible en: https://pubmed.ncbi.nlm.nih.gov/32747912/ doi: 10.47162/RJME.61.1.23 


\section{5.}

Oprinca GC, Muja LA. Postmortem examination of three SARS-CoV-2-positive autopsies including histopathologic and immunohistochemical analysis. Int J Legal Med. [Internet]. 2021 [citado 15 enero 2021];135(1):329-39. Disponible en: https://pubmed. ncbi.nlm.nih.gov/32851474/ doi: 10.1007/s00414020-02406-w.

\section{6.}

Keresztesi AA, Perde F, Ghita-Nanu A, Radu CC, Negrea M, Keresztesi G. Post-mortem diagnosis and autopsy findings in SARS-CoV-2 infection: forensic case series. Diagnóstics [Internet]. 2020 [citado 11 junio 2021];10(12):1070. Disponible en: https://www. ncbi.nlm.nih.gov/pmc/articles/PMC7764537/ doi: 10.3390 / diagnostics10121070.

\section{7.}

Beigmohammadi MT, Jahanbin B, Safaei M, Amoozadeh L, Khoshavi M, Mehrtash V, et al. Pathological findings of postmortem biopsies from lung, heart, and liver of 7 deceased COVID-19 patients. Int J Surg Pathol. [Internet]. 2020 [citado 15 enero 2021];29(2):135-45. Disponible en: https://pubmed.ncbi.nlm.nih.gov/32552178/ doi: 10.1177/1066896920935195.

\section{8.}

Xu Z, Shi L, Wang Y, Zhang J, Huang L, Zhang C, et al. Pathological findings of COVID-19 associated with acute respiratory distress syndrome. Lancet Respir Med [Internet]. 2020 [citado 15 de enero 2021];8(4):420-22. Disponible en: https://pubmed. ncbi.nlm.nih.gov/32085846/

\section{9.}

Qiu Y, Wu D, Ning W, Xu J, Shu T, Huang M, et al. Postmortem tissue proteomics reveals the pathogenesis of multi-organ injuries of COVID-19. Natl Sci Rev [Internet]. 2020 [citado 15 de enero 2021];8(11):nwab143 23. Disponible en: https://pubmed.ncbi.nlm.nih. gov/34876996/ doi: 10.1016/S2213-2600(20)30076-X

\section{0.}

Youd E, Moore L. COVID-19 autopsy in people who died in community settings: the first series. J Clin Pathol [Internet]. 2020 [citado 15 enero 2021];73(12):840844. Disponible en: https://pubmed.ncbi.nlm.nih. gov/32605920/ doi: 10.1136/jclinpath-2020-206710

\section{1.}

Hanley B, Naresh KN, Roufosse C, Nicholson AG, Weir $\mathrm{J}$, Cooke G, et al. Histopathological findings and viral tropism in UK patients with severe fatal COVID-19: a post-mortem study. The Lancet Microbe [Internet]. 2020 [citado 15 enero 2021]; 1(6):e245-e253. Disponible en: https://pubmed.ncbi.nlm.nih. gov/32844161/ doi: 10.1136/jclinpath-2020-206710.

\section{2.}

Varga Z, Flammer A, Steiger P, Haberecker M, Andermatt R, Zinkernagel AS, et al. Endothelial cell infection and endotheliitis in COVID-19. Lancet [Internet]. 2020[citado 15 enero 2021]; 395(10234):1417-1418. Disponible en: https:// pubmed.ncbi.nlm.nih.gov/32325026/ doi: 10.1016/ S0140-6736(20)30937-5 


\section{8.}

Suess C, Hausmann R. Gross and histopathological pulmonary findings in a COVID-19 associated death during self-isolation. Int J Legal Med [Internet]. 2020 [citado 15 enero 2021]; 134(4):1285-1290. Disponible en: https://pubmed.ncbi.nlm.nih.gov/32504146/ doi: 10.1007/s00414-020-02319-8

\section{4}

Santana MF, Silva Borba MG, Baía da Silva DC, Val F, Araujo Alexandre MA, Brito Sousa JD, et al. Case Report: Adrenal pathology findings in severe COVID-19: an autopsy study. Am J Trop Med Hyg [Internet]. 2020 [citado 15 enero 2021];103(4): 16041607. Disponible en: https://pubmed.ncbi.nlm.nih. gov/32876012/ doi: 10.4269/ajtmh.20-0787.

\section{5.}

Adachi T, Chong JM, Nakajima N, Sano M, Yamazaki J, Miyamotoet I, et al. Clinicopathologic and immunohistochemical findings from autopsy of patient with COVID-19, Japan. Emerg Infect Dis [Internet]. 2020 [citado 15 enero 2021]; 26(9): 21572161. Disponible en: https://pubmed.ncbi.nlm.nih. gov/32412897/ doi: 10.3201/eid2609.201353

\section{6.}

Ducloyer M, Gaborit B, Toquet C, Castain L, Bal A, Arrigoni PP, et al. Complete post-mortem data in a fatal case of COVID-19: clinical, radiological and pathological correlations. Int J Legal Med[Internet]. 2020 [citado 15 enero 2021]; 134(6):2209-2214. Disponible en: https://pubmed.ncbi.nlm.nih. gov/32767018/ doi: 10.1007/s00414-020-02390-1

\section{7.}

Schwensen HF, Borreschmidt LK, Storgaard M, Redsted S, Christensen S, Madsen LB. Fatal pulmonary fibrosis: a post-COVID-19 autopsy case. J Clin Pathol [Internet]. 2020 [citado 15 enero 2021]; 206879. Disponible en: https://pubmed.ncbi.nlm.nih.gov/32723800/ doi: 10.1136/jclinpath-2020-206879

\section{8.}

Valdivia Mazeira MF, Salas C, Nieves Alonso JM, Martín Fragueiro L, Bárcena C, Muñoz Hernandez P, et al. Increased number of pulmonary megakaryocytes in COVID-19 patients with diffuse alveolar damage: an autopsy study with clinical correlation and review of the literature. Virchows Arch [Internet]. 2020 [citado 15 enero 2021]; 478(3): 487-496. Disponible en: https://pubmed.ncbi.nlm.nih.gov/32915265/ doi: 10.1007/s00428-020-02926-1

\section{9.}

Porembskaya O., Lobastov K., Pashovkina O., Tsaplin S., Schastlivtsev I, Zhuravlev S, et al. Thrombosis of pulmonary vasculature despite anticoagulation and thrombolysis: the findings from seven autopsies. Thrombosis Update[Internet]. 2020 [citado 15 enero 2021]; 1:100017. Disponible en: https:/www.sciencedirect.com/science/article/pii/ S2666572720300171 doi: 10.1016/j.tru.2020.100017.

\section{0.}

Zhou B, Zhao W, Feng R, Zhang X, Li X, Zhou Y, et al. The pathological autopsy of coronavirus disease 2019 (COVID-2019) in China: a review. Pathog Dis[Internet]. 2020 [citado 15 enero 2021]; 78(3): ftaa026. Disponible en: https://pubmed.ncbi.nlm.nih. gov/32533838/ doi: 10.1093/femspd/ftaa026 


\section{1.}

Bian XW, Yao XH, Ping YF, Yu S, Shi Y, Luo T, et al. Autopsy of COVID-19 patients in China. National Science Review [Internet]. 2020 [citado 15 enero 2021]; 7(9):1414-1418. Disponible en: https://www. researchgate.net/publication/345454017_Autopsy_ of_COVID-19_patients_in_China doi: 10.1093/nsr/ nwaa123

\section{2.}

Barría C. Coronavirus: los 10 países que más han gastado en enfrentar la pandemia: (y cómo se ubican los de América Latina). [Internet]. Estados Unidos: BBC News Mundo; 2020. [Actualizado 18 mayo 2020; citado 15 enero 2021]. Disponible en: https:// www.bbc.com/mundo/noticias-52686453

\section{3.}

Salerno M, Sessa F, Piscopo A, Montana A, Torrisi M, Patanè F, et al. No Autopsies on COVID-19 deaths: a missed opportunity and the lockdown of science. J Clin Med[Internet]. 2020 [citado 15 enero 2021]; 9(5): 1472. Disponible en: https://pubmed.ncbi.nlm.nih. gov/32422983/ doi: 10.3390/jcm9051472

\section{4.}

Naciones unidas. El impacto del COVID-19 en América Latina y el Caribe [Internet]. Nueva York: GNUDS; 2020. [citado 15 enero 2021]. Disponible en: https://unsdg.un.org/es/resources/resumen-depoliticas-el-impacto-de-covid-19-en-america-latinay-el-caribe

\section{5.}

Aquila I, Sacco MA, Abenavoli L, Malara N, Arena V, Grassi S. et al. Severe acute respiratory syndrome Coronavirus 2 Pandemic. Arch Pathol Lab Med[Internet]. 2020 [citado 15 enero 2021]; 144(9):1048-1056. Disponible en: https://pubmed. ncbi.nlm.nih.gov/32383963/ doi: 10.5858/arpa.20200165-SA

\section{6.}

Siefring C, Sachire J, Thomas D, Allenby P, Exposure reduction in COVID-19 autopsies. Autops Case Rep [Internet]. 2020 [citado 15 enero 2021]; 10(3):e2020193. Disponible en: https://pubmed.ncbi.nlm.nih. gov/33344303/ doi: 10.4322/acr.2020.193

\section{7.}

Khatam Lashgari A, Henningsen MJ, Olsen KB, Jacobsen C, Hasselby JP, Colville Ebeling B, et al. Autopsies in pandemics - a perspective on barriers and benefits. Is it time for a revival?. APMIS [Internet]. 2021 [citado 11 julio 2021]; 129(7): 324339. Disponible en: https://onlinelibrary.wiley.com/ doi: https://doi.org/10.1111/apm.13111

\section{8.}

Espinosa Brito AD, de Mendoza Amat JH. In defense of clinical autopsy and Its practice in Cuba. MEDICC Review [Internet]. 2017 [citado 11 julio 2021]; 19(1):37-41. Disponible en: https://www.redalyc.org/ articulo.oa? $\mathrm{id}=437549486007$ 


\section{9.}

Ong BB, Milne N. Limited post-mortem examination. An alternative and viable way to avoid full examination? Forensic Sci Med Pathol [Internet]. 2007[citado 11 julio 2021];3(3):188-193. Disponible en: https://pubmed.ncbi.nlm.nih.gov/25869162/ doi: 10.1007/s12024-007-0021-6.

\section{0.}

Luongo de Matos LL, Trufelli DC, Luongo de Matos MG, da Silva Pinhal MA. Immunohistochemistry as an important tool in biomarkers detection and clinical practice. Biomark Insights[Internet]. 2010[citado 15 enero 2021];5:9-20. Disponible en: https://pubmed. ncbi.nlm.nih.gov/20212918/ doi: 10.4137/bmi.s2185

\section{1.}

Buitrago Sierra R, Guzmán Á, Santa Marin JF. Caracterización morfológica del SARS-CoV-2 mediante microscopía electrónica. TecnoL [Internet]. 2021[citado 15 enero 2021];24(50): e1675. Disponible en: http://www.scielo.org.co/scielo.php?script=sci arttext\&pid=S0123-77992021000100257 . doi: https://doi.org/10.22430/22565337.1675

\section{2.}

Serrano N, Páez Leal MC, Luna Gonzales M, Guío Mahecha E. Biobanco: herramienta fundamental para la investigación biomédica actual. Rev Univ Ind Santander Salud [Internet] . 2016 [citado 15 enero 2021];48(1): 97-117. Disponible en: https://www. researchgate.net/publication/295098379_Biobanco_ Herramienta_fundamental_para_la_investigacion_ biomedica_actual doi:10.18273/revsal.v48 n12016011

\section{3.}

Mohanty SK, Satapathy A, Naidu MM, Mukhopadhyay S, Sharma S, Barton LM, et al. Severe acute respiratory syndrome coronavirus-2 (SARS-CoV-2) and coronavirus disease 19 (COVID-19): anatomic pathology perspective on current knowledge. Diagn Pathol [Internet]. 2020 [citado 15 enero 2021]; 15(1):103. Disponible en: https://pubmed.ncbi.nlm. nih.gov/32799894/ doi: 10.1186/s13000-020-010178.

\section{4.}

Sessa F, Salerno M, Pomara C. Autopsy tool in unknown diseases: the experience with Coronaviruses (SARSCoV, MERS-CoV, SARS-CoV-2). Medicina[Internet]. 2021 [citado 2021 julio 10]; 57(4): 309. Disponible en: https:/www.mdpi.com/1648-9144/57/4/309\#cite https://doi.org/10.3390/medicina57040309

\section{5.}

Zayratyants OV, Samsonova MV, Cherniaev AL, Mishnev OD, Mikhaleva LM, Krupnov NM, et al. Patología COVID-19: experiencia de 2000 autopsias. Rev Rusa Med Forense [Internet]. 2020 [citado 2021 julio 10]; 6(4): 10-23. Disponible en: https://for-medex. ru/jour/article/view/340 doi: https://doi.org/10.19048/ fm340

\section{6.}

Sperhake JP. Autopsies of COVID-19 deceased? Absolutely! Leg Med (Tokyo) [Internet]. 2020 [citado 2021 julio 10]; 47:101769. Disponible en: https:// www.ncbi.nlm.nih.gov/pmc/articles/PMC7382931/ doi: 10.1016/j.legalmed.2020.101769 


\section{7.}

Jain U. Effect of COVID-19 on the organs. Cureus[Internet]. 2020 [citado 15 enero 2021]; 12(8): e9540. Disponible en: https://pubmed.ncbi.nlm.nih. gov/32905500/ doi: 10.7759/cureus.9540

\section{8.}

Aquino Canchari CR, Quispe Canchari CR, Huaman Castillon KM. COVID-19y su relacióncon poblaciones vulnerables. Rev haban cienc méd [Internet]. 2020 [citado 15 enero 2021];19:1-18. Disponible en: $\quad h t t p: / / s c i e l o . s l d . c u / s c i e l o . p h p ? s c r i p t=s c i$ arttext\&pid=S1729-519X2020000400005\#B16

\section{9.}

Naciones Unidas. Informe de políticas: los efectos de la covid-19 en las personas de edad; [Internet]. New York: ONU; 2020. [actualizado mayo 2020]. Disponible en: https://www.un.org/sites/un2.un.org/ files/old_persons_spanish.pd

\section{0.}

Organización mundial de la salud. El género y la COVID-19: documento de apoyo, 14 de mayo de 2020[Internet]. Ginebra: OMS; 2020. [citado 15 enero 2021]. Disponible en: https://apps.who.int/iris/ handle/10665/332242

\section{1.}

Salinas Aguirre JE, Sánchez García C, Rodríguez Sanchez R, Rodríguez Muñoz L, Díaz Castaño A, Bernal Gómez R. Características clínicas y comorbilidades asociadas a mortalidad en pacientes con COVID-19 en Coahuila (México). Rev Clín Esp [Internet]. 2021 [citado 15 enero 2021]. Disponible en: https://www.ncbi.nlm.nih.gov/pmc/articles/ PMC7816557/ doi: 10.1016/j.rce.2020.12.006

\section{2.}

Guan WJ, Liang WH, Zhao Y, Liang HR, Chen ZS, Li YM et al. Comorbidity and its impact on 1,590 patients with COVID-19 in China: a nationwide analysis. medRxiv [Preprint]. 2020 [citado 15 enero 2021].Disponible en: https://doi.org/10.1101/2020.02 .25 .20027664 .

\section{3.}

Currie D, O'Brien K, Phillips O, St Jean M, Turcotte M, Willbond S, et al. COVID-19 death comorbidities in Canada. Canada: Statistics Canada; 2020 [citado 15 enero 2021]. Disponible en: https://www150.statcan. gc.ca/n1/en/catalogue/45280001202000100087

\section{4.}

Organización Panamericana de la salud, Organización mundial de la salud. Orientación internacional para la certificación y clasificación (codificación) del COVID-19 como causa de muerte. [Internet]. Ginebra: OPS, OMS; 2020. [citado 20 de abril de 2020]. Disponible en: https://www.paho.org/es/documentos/ orientacion-internacional-para-certificacionclasificacion-codificacion-covid-19-como

\section{5.}

Hirschbühl K, Schaller T, Kling E, Märkl B, Claus R. Autopsy of patients with COVID-19: a balance of fear and curiosity. Pathol Res Pract [Internet]. 2020 [citado 15 enero 2021] ; 216(8):153039. Disponible en: https://pubmed.ncbi.nlm.nih.gov/32703502/ doi: 10.1016/j.prp.2020.153039 


\section{6.}

Centers for disease control and prevention. Collection and Submission of Postmortem Specimens from Deceased Persons with Confirmed or Suspected COVID-19: Postmortem Guidance. Atlanta(US): CDC; 2020. [actualizado 2 dic. 2020; citado 22 febrero 2021]. Disponible en: https://www.cdc.gov/ coronavirus/2019-ncov/hcp/guidance-postmortemspecimens.html

\section{7.}

Hasmi AH, Khoo LS, Koo ZP, Suriani MUA, Hamdan AN, Yaro SWM. et al. The craniotomy box: An innovative method of containing hazardous aerosols generated during skull saw use in autopsy on a COVID-19 body. Forensic Sci Med Pathol [Internet]. 2020 [citado 15 enero 2021];16(3):477-80. Disponible en: https://pubmed.ncbi.nlm.nih.gov/32500339/ doi: 10.1007/s12024-020-00270-z.

\section{8.}

PrietoCarreroJ,FondebriderL, Salado PuertoM,Tidball Binz M. La gestión de las personas fallecidas a causa de la pandemia de COVID-19 y los retos organizativos desde la óptica de los servicios medicolegales. Rev Española Med Legal [Internet]. 2021[citado 15 enero 2021];47(4): 164-171. Disponible en: https://www. ncbi.nlm.nih.gov/pmc/articles/PMC7825931/D Doi: 10.1016/j.reml.2021.01.003

\section{9.}

Gonzalez E, Harrison C, Hopkins K, Horwitz L, Nagovitch P, Sonneland HK, et al. El coronavirus en América Latina. [Internet] New York: Americas Society,Council of the Americas; 2021. [citado 15 junio 2021]. Disponible en: https://www.as-coa.org/ articles/el-coronavirus-en-america-latina

\section{0.}

Rios Valencia J, Gamboa Domínguez A. Coupe de Grace: autopsy in COVID-19 Pandemic. Rev Invest Clin[Internet]. 2020 [citado 15 enero 2021];72(5). Disponible en: https://pubmed.ncbi.nlm.nih. gov/33053577/ doi: 10.24875/RIC.20000262

\section{1.}

Honduras. Congreso Nacional, Corte Suprema de Justicia. Ley de Autopsia Medica Obligatoria, Decreto Numero 182-84. Diario Oficial La Gaceta No. 24486 [Internet]. 5 de diciembre de 1984. [citado 15 enero 2021]. Disponible en: https://www.poderjudicial.gob. hn/CEDIJ/Documents/Ley\%20de\%20Autopsia\%20 Medica\%20Obligatoria.pdf 